\title{
ON THE GEOCHEMISTRY OF KAATIALA PEGMATITE, WESTERN FINLAND
}

\author{
KALERVO NIEIMINEN *
}

\begin{abstract}
NIEMINEN, KALERVO 1978: On the geochemistry of Kaatiala pegmatite, western Finland. Bull. Geol. Soc. Finland 50: 1-14.

Spark source mass spectrometry with electrical detection was used for determining 36 elements in 25 individual mineral samples, bulk samples and country rock samples from the Kaatiala pegmatite deposit in Finland. Also determined were the sum of the REE in all the samples, and the distribution pattern in the samples with higher REE contents.

The mineralogical and chemical balance of the whole pegmatite was estimated on the basis of the analytical data.
\end{abstract}

Kalervo Nieminen, Department of Chemistry, Helsinki University of Technology, SF-02150 Espoo 15, Finland.

\section{History}

The Kaatiala pegmatite, located in the parish of Kuortane, W. Finland (Fig. 1) was first mentioned by Holmberg (1857). The first detailed geological description was given by Laitakari (1914).

The exploitation of the pegmatite started in 1942. Before then only quartz had been temporarily quarried at the end of the last century. However, the needs of the steelmaking industry during the Second World War made it feasible to reopen the mine. Soon afterwards, the production of feldspar for the domestic ceramics industry was also started up. Quarrying was initially directed at the quartz and feldspar core of the deposit.

Wartime conditions meant that there was an acute shortage of all kinds of equipment. Both drilling and the sorting of blasted rock were done by hand. The sorted products were carried on stretchers from the bottom of the quarry to stock piles at the edge of the pit.

When enough material had been produced, an application was made to the Ministry of Trade and Industry for a special licence to transport the products by truck to Niinimaa railway station, $10 \mathrm{~km}$ to the west.

The quarrying was gradually modernized. In 1943 , the stretchers were replaced by a hand windlass. In 1945-1947 a steam winch, donkey pumps and a locomotive-driven compressor were installed. In 1950-1951 the steam engines were replaced by electric motors, and in 1951 the first sorting plant was built. This consisted of a wooden silo from which the rock ran on to sorting tables arranged radially around the silo. On the tables pure feldspar and quartz were sorted 


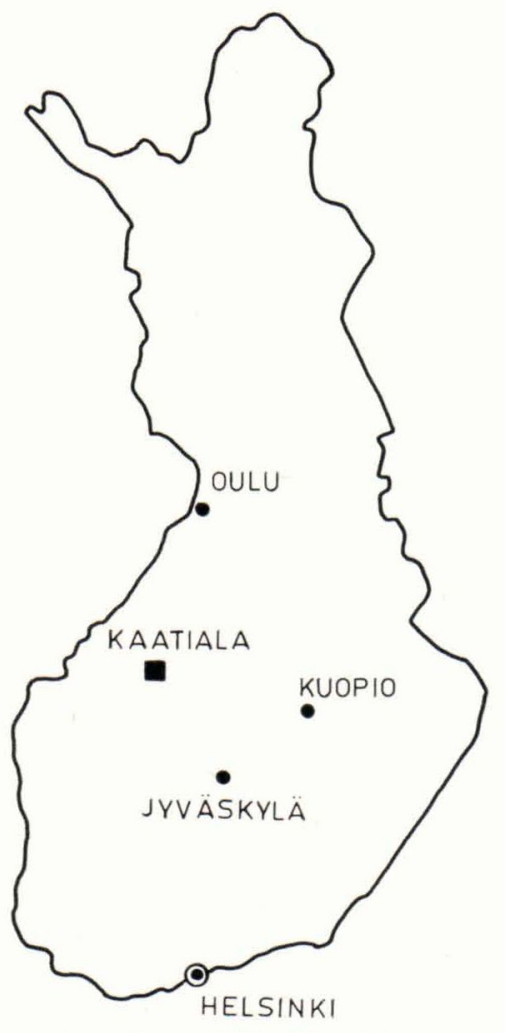

Fig. 1. Location of the Kaatiala pegmatite.

out from the gangue. Rock fragments consisting of several minerals were hammered down in size until monomineralic feldspar or quartz was obtained.

The last version of the sorting plant was built in 1956 (Fig. 2). The rock was crushed by jaw crusher under $75 \mathrm{~mm}$ and screened into three fractions: $75-50 \mathrm{~mm}, 50-25 \mathrm{~mm}$, and minus $25 \mathrm{~mm}$. The minus $25 \mathrm{~mm}$ fraction went directly to waste. The coarse fractions proceeded to the sorting belts where the monomineralic quartz, feldspar and other mineral products were picked out and dropped each into their own silo.

With the modernization of the quarrying and sorting, however, it became increasingly more difficult to recover the valuable minerals. Quarrying operations had first centered on the core zone, which consisted of huge quartz and potassium feldspar crystals. Sorting was easy and produced no waste at all. Over the years, however, it became necessary to mine more and more heterogeneous zones with small crystal size and ever larger amounts of tourmaline and other impurities.

To obtain first class products but with low recovery, all lumps containing tourmaline or quartz had to be discarded, no matter how low the content of these minerals was, or else a more impure product with higher recovery had to be accepted. A compromise was reached and in addition to the first-class lump product, a second-class product with some tourmaline and quartz inclusions was also accepted. This product was further milled and cleaned by a high intensity

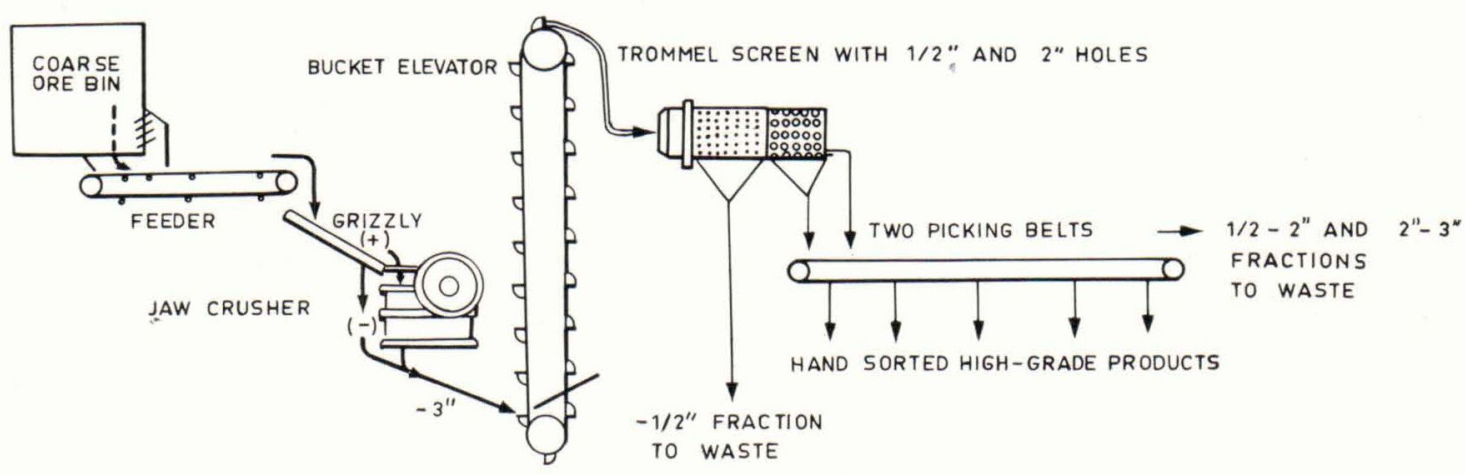

Fig. 2. Flowsheet of the Kaatiala sorting plant. 
Table 1. Production statistics of Kaatiala pegmatite from 1942 to 1969.

Quarried

Pegmatite

516.000 tons

Gangue

136.000 tons

Produced

Potassium feldspar

Quartz

Mica

Beryl

Columbite

Löllingite

Wasted

$0-25 \mathrm{~mm}$ fraction

$>25 \mathrm{~mm}$ waste

134.000 tons 193.000 tons

magnetic separator in the Tapanila feldspar mill near Helsinki. It also became expedient to mine the gangue on the hanging wall to an ever increasing extent.

In the final operational years the ratio of gangue to pegmatite exceeded 1. Mining became unprofitable and in 1968 was stopped altogether. A total of 516000 tons of pegmatite and 136000 tons of gangue had been mined. Statistics of the production are presented in Table 1.

\section{Geology and mineralogy}

The geology of Kaatiala deposit, based mainly on the works of Tavela (1949), Volborth (1952), Neuvonen (1960), and on the author's own observations during the quarrying period, is presented in Fig. 3. The pegmatite lens dips about 15 degrees to NE through granodioritic country rock. The border zone closest to the footwall was characterized by sodium feldspar, whereas the border zone on the hanging wall was an

\section{EXPLANATION}

$3^{2} 2^{2}$ Granodioritic country rock

$\Delta \square^{\Delta}$ Quartz( $\left.\Delta\right)$-microcline (0) pegmotite

$\Delta / 0$ Quartz( $\Delta)$-microcline $(0)$ core

Albite (11)-muscovite (1) pegmatite

$\begin{array}{llll}\text { As Löllingite } & \text { Sp Spodumene } \\ \text { Ap Apotite } & \text { TT } \text { Tourmaline } \\ \text { Be Beryl } & \text { GT Green tourmaline } \\ \text { Cb } & \text { Columbite } & \text { RT } & \text { Red tourmaline } \\ \text { Sn Cossiterite } & \odot & \text { Drill hole } \\ \therefore \text { Quarry boundary } & \end{array}$
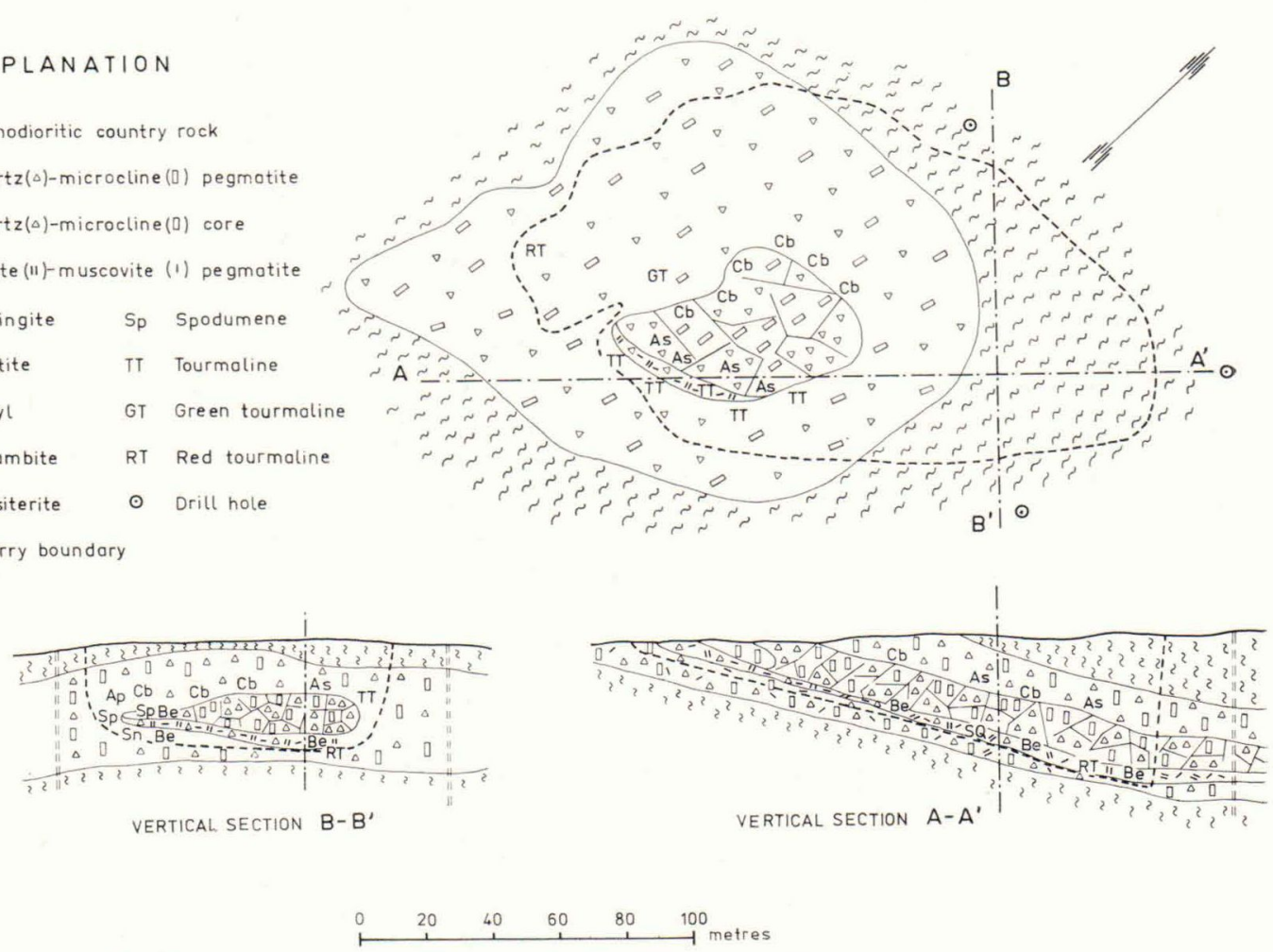

Fig. 3. Geology of the Kaatiala feldspar quarry. $S Q=$ smoky quartz. 
aplitic variety of pegmatite, which after 2 to 5 metres graded into medium- or coarsegrained microcline-quartz-muscovite pegmatite with black tourmaline. On the southwestern side, nearest to the centrally located intermediate and core zones, there was a nearly vertical, roughly $5 \mathrm{~m}$ wide zone with very abundant tourmaline. The central parts were underlain by muscovite-rich zones with beryl. Löllingite, columbite and spodumene occurred mainly above the central part.

The central part consisted of large microcline crystals and discrete quartz cores, with some rose quartz and smoke quartz crystals. Cavities with transparent green and red tourmalines, water-clear beryls and lepidolite were encountered near the narrow northwestern margin. This same zone also contained spodumene, and the only cassiterite specimen found in the deposit.

Feldspars occurred in Kaatiala as microcline with 15 to $22 \mathrm{~mole}-\%$ albite, and as plagioclase with 12 mole- $\%$ anorthite. No transitional forms in larger quantities were met with. The two feldspar varieties differed conspicuously in appearence. The potassium feldspar crystals were grey in colour with clear lustrous faces, whereas the sodium feldspar was white in colour with mat and dull surfaces. Thus, even hand sorting could produce relatively pure products in terms of the ratio of potassium to sodium.

\section{Chemical analyses}

The chemical composition of Kaatiala pegmatite was investigated by supplementing the analyses made during the operational period with new multi-element analyses of the various minerals, of the representative bulk samples, and of the country rock.

Analyses of the main components for pure microcline and albite specimens from the operational period as well as partial analyses
Table 2. Composition of pure feldspar lumps.

\begin{tabular}{|c|c|c|c|}
\hline \multicolumn{4}{|c|}{ Chemical analyses a } \\
\hline & $\%$ & $0 \%$ & $\%$ \\
\hline $\mathrm{SiO}_{2}$ & 65.31 & 63.73 & 64.79 \\
\hline $\mathrm{TiO}_{2}$ & 0.00 & 0.00 & 0.00 \\
\hline $\mathrm{Al}_{2} \overrightarrow{\mathrm{O}}_{3}$ & 18.81 & 18.99 & 21.80 \\
\hline $\mathrm{Fe}_{2} \mathrm{O}_{3}$ & 0.04 & 0.07 & 0.11 \\
\hline $\mathrm{MnO}$ & 0.00 & 0.00 & 0.01 \\
\hline $\mathrm{MgO}$ & 0.03 & 0.01 & 0.02 \\
\hline $\mathrm{CaO}$ & 0.04 & 0.07 & 2.51 \\
\hline $\mathrm{Li}_{2} \mathrm{O}$ & 0.02 & 0.02 & 0.02 \\
\hline $\mathrm{Na}_{2} \mathrm{O}$ & 2.46 & 1.70 & 9.66 \\
\hline $\mathrm{K}_{2} \mathrm{O}$ & 12.99 & 14.02 & 0.69 \\
\hline $\mathrm{Rb}_{2} \mathrm{O}$ & 0.12 & 0.33 & 0.00 \\
\hline $\mathrm{Cs}_{2} \mathrm{O}$ & 0.03 & 0.20 & 0.00 \\
\hline $\mathrm{P}_{2} \mathrm{O}_{5}$ & 0.13 & 0.70 & 0.04 \\
\hline $\mathrm{H}_{2} \mathrm{O}+$ & 0.23 & 0.14 & 0.28 \\
\hline $\mathrm{H}_{2} \mathrm{O}-$ & 0.05 & 0.12 & 0.05 \\
\hline Totals & 100.26 & 100.10 & 99.98 \\
\hline \multicolumn{4}{|c|}{ Norms ${ }^{b}$} \\
\hline $\mathrm{Q}$ & 0.7 & 0.0 & 0.5 \\
\hline Or & 76.2 & 83.8 & 3.9 \\
\hline $\mathrm{Ab}$ & 21.9 & 15.2 & 82.9 \\
\hline An & 0.2 & 0.4 & 11.9 \\
\hline Totals & 99.0 & 99.4 & 99.2 \\
\hline
\end{tabular}

a Analyst: P. Ojanperä, Geological Survey of Finland.

b Method after Hutchison (1974).

for feldspar concentrates with norm values for quartz, ortoclase, albite and anortite are presented in Tables 2 and 3 .

The minerals selected for multi-element analysis were typical pure specimens of the most common types. The bulk samples were taken from stock piles of the minus $25 \mathrm{~mm}$ fraction, obtained by screening the crushed pegmatite before hand sorting. Its chemical composition closely approximates the composition of the whole pegmatite body.

The first country rock sample was taken from the $\mathrm{E}$ side and the second from $\mathrm{N}$ side of the pegmatite at a distance of 25 metres from the contact.

The analyses were performed mainly with an MS 702 double focusing spark source mass spectrometer using electrical detection. The 
Table 3. Composition of Kaatiala feldspar concentrates.

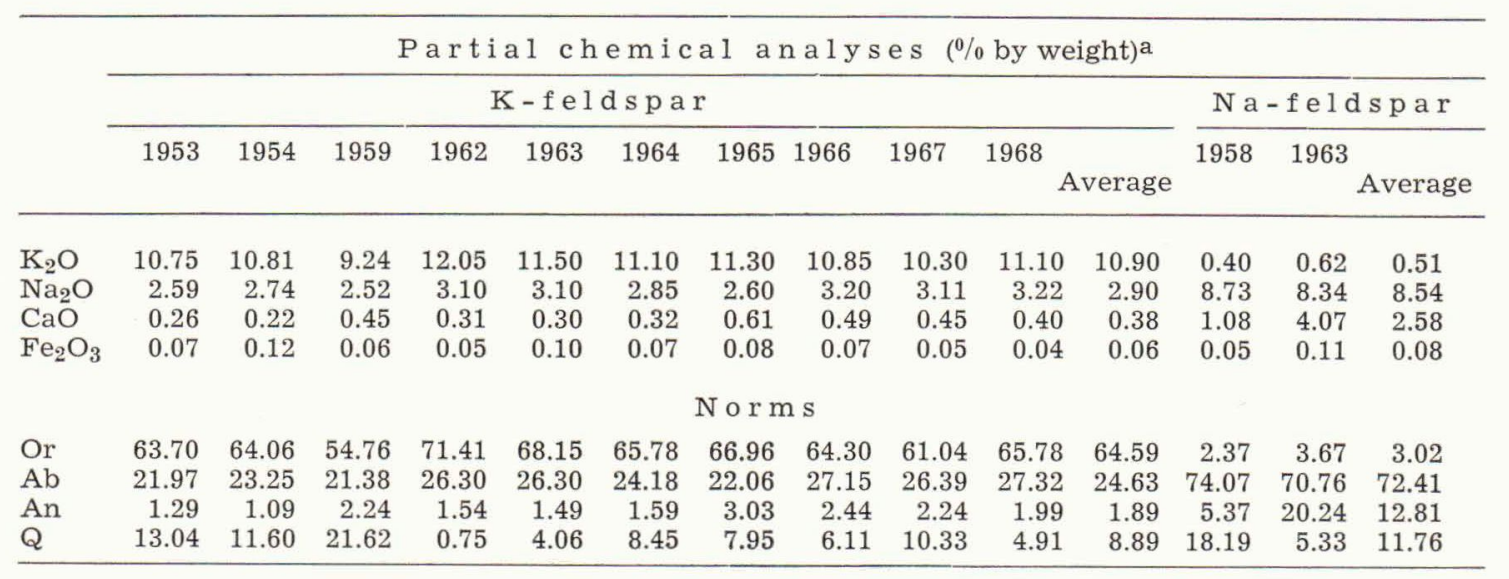

a Each sample represents a carload (16.5 tons) milled and analysed at Tapanila feldspar mill.

analytical procedure has been described by Nieminen and Yliruokanen (1974). In addition to the ratios peak height/content ppm in weight, which constituted the basis for calculating the contents of certain elements, some additional values were calculated with the aid of a third standard, a Limestone ZG1$\mathrm{KH}$ (Flanagan 1973). The new ratios are presented in Table 4.

Table 4. The ratios peak height/content ppm by weight in ZGl-KH

\section{Isotope}

$\begin{array}{cc}{ }^{33} \mathrm{~S}^{+} & 0.4 \\ 53 \mathrm{Cr}^{+}+ & 0.14 \\ 61 \mathrm{Ni}^{+} & 0.40 \\ 62 \mathrm{Ni}^{+} & 1.15 \\ 67 \mathrm{Zn}^{+} & 0.82 \\ 67 \mathrm{Zn}^{+}+ & 0.10 \\ 71 \mathrm{Ga}^{+} & 3.38 \\ 71 \mathrm{Ga}^{+} & 0.15 \\ 73 \mathrm{Ge}^{+} & 0.63 \\ 75 \mathrm{As}^{+} & 4.42 \\ 77 \mathrm{Se}^{+} & 27.4 \\ 88 \mathrm{Sr}^{+} & 3.0 \\ 92 \mathrm{Zr}+ & 0.26 \\ 121 \mathrm{Sb}^{+} & 1.21 \\ 123 \mathrm{Sb}^{+} & 1.35 \\ 182 \mathrm{~W}^{+} & 0.93 \\ 184 \mathrm{~W}^{+} & 1.08 \\ 209 \mathrm{Bi}^{+}+ & 0.37\end{array}$

Because the contents of lanthanoids and barium were low, it was possible in this work to use the following isotopes: ${ }^{67} \mathrm{Zn},{ }^{71} \mathrm{Ga}$, ${ }^{73} \mathrm{Ge}$, and ${ }^{75} \mathrm{As}$ for the calculations. Further, the standard ZGI-KH is also more convenient for determining the isotopes ${ }^{33} \mathrm{~S},{ }^{61} \mathrm{Ni},{ }^{62} \mathrm{Ni}$, ${ }^{75} \mathrm{As},{ }^{77} \mathrm{Se},{ }^{88} \mathrm{Sr},{ }^{92} \mathrm{Zr},{ }^{114} \mathrm{In},{ }^{123} \mathrm{Sb},{ }^{183} \mathrm{~W},{ }^{184} \mathrm{~W}$, and ${ }^{209} \mathrm{Bi}$ than the standards USGS-G-1 and USGS-W-1. AAS replaced mass spectrometry (Bernas 1968) in those cases where high contents of alkalies and beryllium were present.

Three chemical analyses of beryl have already been published by Erämetsä et al. (1973). The new chemical analyses are presented in Tables $5 \mathrm{a}$ and $5 \mathrm{~b}$.

\section{Mineralogical and chemical balance}

With the aid of the production figures (Table 1) and of the analyses, Tables 2, 3, 5a, and $5 \mathrm{~b}$ an attempt was made to calculate the total amounts of the various minerals of the Kaatiala pegmatite, Table 6. In calculations, the chemical composition of the bulk sample of the minus $25 \mathrm{~mm}$ fraction was taken to represent the whole pegmatite body. The 
Table 5 a. Multielement analyses of Kaatiala minerals and rocks. The contents are given as ppm.

- Not determined < below detection limit

\begin{tabular}{|c|c|c|c|c|c|c|c|c|c|c|c|c|c|c|c|c|c|c|c|c|}
\hline \multirow[b]{2}{*}{ No. } & \multirow[b]{2}{*}{ Sample } & \multicolumn{2}{|c|}{ Group 1} & \multicolumn{8}{|c|}{ Group 2} & \multicolumn{7}{|c|}{ Group 3} & \multicolumn{2}{|c|}{ Group 4} \\
\hline & & $\mathrm{Be}$ & B & $\mathrm{Li}$ & $\mathrm{Na}$ & $\mathrm{K}$ & $\mathrm{Rb}$ & Cs & $\mathrm{Ca}$ & $\mathrm{Sr}$ & $\mathrm{Ba}$ & $\mathrm{Cu}$ & $\mathrm{Zn}$ & $\mathrm{Ga}$ & $\mathrm{Ge}$ & As & $\mathrm{Tl}$ & $\mathrm{Pb}$ & $\mathrm{Y}$ & REE \\
\hline $\begin{array}{c}28 \\
2 \\
16 \\
33 \\
15\end{array}$ & $\begin{array}{l}\text { Microcline perthite } \\
\text { Microcline perthite } \\
\text { Na-rich microcline } \\
\text { Microcline, transp. } \\
\text { Black microcline }\end{array}$ & $\begin{array}{r}2 \\
3 \\
7 \\
4\end{array}$ & $\begin{array}{r}3 \\
5 \\
4.5 \\
7 \\
4\end{array}$ & $\begin{array}{r}20 \\
50 \\
25 \\
700 \\
40\end{array}$ & $\begin{array}{r}15000 \\
17000 \\
70000 \\
4000 \\
16000\end{array}$ & $\begin{array}{r}91500 \\
105000 \\
56000 \\
125000 \\
95000\end{array}$ & $\begin{array}{r}300 \\
800 \\
450 \\
2500 \\
900\end{array}$ & $\begin{array}{r}35 \\
20 \\
45 \\
800 \\
50\end{array}$ & $\begin{array}{l}1500 \\
2000 \\
1800 \\
1300 \\
2000\end{array}$ & $\begin{array}{r}50 \\
6 \\
20 \\
5 \\
12\end{array}$ & $\begin{array}{r}120 \\
10 \\
50 \\
40 \\
60\end{array}$ & $\begin{array}{r}1.0 \\
6 \\
22 \\
7 \\
30\end{array}$ & $\begin{array}{l}25 \\
15 \\
20 \\
20 \\
30\end{array}$ & $\begin{array}{l}15 \\
20 \\
12 \\
20 \\
25\end{array}$ & $\begin{array}{l}1.0 \\
1.0 \\
4.7 \\
10 \\
4.6\end{array}$ & $\begin{array}{r}1.0 \\
5 \\
1.7 \\
30 \\
410\end{array}$ & $\begin{array}{r}2.2 \\
5 \\
3.8 \\
20 \\
7\end{array}$ & $\begin{array}{r}100 \\
50 \\
90 \\
30 \\
450\end{array}$ & $\begin{array}{l}< \\
\sum \\
1.2\end{array}$ & $\begin{array}{r}\vdots \\
\vdots \\
4 \\
14\end{array}$ \\
\hline $\begin{array}{l}18 \\
20\end{array}$ & $\begin{array}{l}\text { Oligoclase } \\
\text { Green albite }\end{array}$ & $\begin{array}{l}1 \\
5\end{array}$ & $\begin{array}{r}0.8 \\
7\end{array}$ & $\begin{array}{l}2.1 \\
14\end{array}$ & $\begin{array}{r}98000 \\
117000\end{array}$ & $\begin{array}{r}600 \\
7200\end{array}$ & $\begin{array}{r}2 \\
14\end{array}$ & $\begin{array}{l}0.5 \\
3.2\end{array}$ & $\begin{array}{r}6700 \\
11000\end{array}$ & $\begin{array}{l}45 \\
20\end{array}$ & $<_{6}$ & $\begin{array}{l}1.9 \\
2.1\end{array}$ & $\begin{array}{r}55 \\
4.8\end{array}$ & $\begin{array}{l}30 \\
30\end{array}$ & $\begin{array}{l}4.0 \\
3.4\end{array}$ & $\begin{array}{r}1.8 \\
60\end{array}$ & $\begin{array}{l}< \\
0.2\end{array}$ & $\begin{array}{l}11 \\
40\end{array}$ & $\begin{array}{r}< \\
18\end{array}$ & 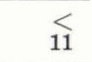 \\
\hline $\begin{array}{r}1 \\
38 \\
37\end{array}$ & $\begin{array}{l}\text { Quartz, regular } \\
\text { Rose quartz } \\
\text { Smoky quartz }\end{array}$ & $\begin{array}{l}0.1 \\
0.5 \\
0.4\end{array}$ & $\begin{array}{l}1.0 \\
1.0 \\
1.0\end{array}$ & $\begin{array}{r}5 \\
40 \\
35\end{array}$ & $\begin{array}{r}5 \\
30 \\
15\end{array}$ & $\begin{array}{r}40 \\
125 \\
130\end{array}$ & $\begin{array}{l}0.2 \\
0.1 \\
0.1\end{array}$ & $\begin{array}{l}0.2 \\
0.5 \\
-\end{array}$ & $\begin{array}{r}110 \\
50 \\
60\end{array}$ & $\begin{array}{l}1.0 \\
0.8 \\
0.5\end{array}$ & 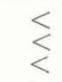 & $\begin{array}{l}0.5 \\
0.5 \\
1.0\end{array}$ & $\begin{array}{l}0.6 \\
1.0 \\
2.5\end{array}$ & $\overline{\bar{z}}$ & $\begin{array}{l}1.7 \\
1.1 \\
3.0\end{array}$ & $\begin{array}{l}0.1 \\
0.3 \\
0.5\end{array}$ & $<$ & $\stackrel{<}{<}$ & $\dot{<}$ & $\stackrel{<}{<}$ \\
\hline $\begin{array}{l}6 \\
7\end{array}$ & $\begin{array}{l}\text { Muscovite } \\
\text { Li-rich mica }\end{array}$ & $\begin{array}{l}10 \\
50\end{array}$ & $\begin{array}{l}70 \\
70\end{array}$ & $\begin{array}{l}1500 \\
7000\end{array}$ & $\begin{array}{l}5000 \\
2000\end{array}$ & $\begin{array}{l}70000 \\
49000\end{array}$ & $\begin{array}{l}1500 \\
3000\end{array}$ & $\begin{array}{r}120 \\
2000\end{array}$ & $\begin{array}{l}1000 \\
1000\end{array}$ & 11 & 1.5 & $\begin{array}{l}5 \\
3\end{array}$ & $\begin{array}{l}30 \\
50\end{array}$ & $\begin{array}{r}65 \\
100\end{array}$ & $\begin{array}{r}5 \\
10\end{array}$ & $\begin{array}{r}10 \\
1.5\end{array}$ & $\begin{array}{r}5 \\
15\end{array}$ & $\stackrel{5}{<}$ & $\begin{array}{r}1.2 \\
6\end{array}$ & $<$ \\
\hline $\begin{array}{r}4 \\
34 \\
35\end{array}$ & $\begin{array}{l}\text { Tourmaline, schorl. } \\
\text { Green tourmaline } \\
\text { Red tourmaline }\end{array}$ & $\begin{array}{r}2 \\
15 \\
11\end{array}$ & $\begin{array}{l}28000 \\
28000 \\
30000\end{array}$ & $\begin{array}{r}50 \\
800 \\
1000\end{array}$ & $\begin{array}{l}12000 \\
21000 \\
18000\end{array}$ & $\begin{array}{r}1200 \\
2300 \\
140\end{array}$ & $\begin{array}{r}5 \\
25 \\
1.2\end{array}$ & $\begin{array}{r}2 \\
120 \\
8\end{array}$ & $\begin{array}{l}1000 \\
1500 \\
1200\end{array}$ & $\begin{array}{r}2.5 \\
6 \\
5\end{array}$ & $\dot{<}$ & $\begin{array}{l}3.3 \\
3.7 \\
2.5\end{array}$ & $\begin{array}{r}700 \\
300 \\
25\end{array}$ & $\begin{array}{l}130 \\
100 \\
350\end{array}$ & $\begin{array}{r}3.4 \\
9 \\
8\end{array}$ & $\begin{array}{l}65 \\
1.8 \\
2.0\end{array}$ & $\stackrel{<}{0.3}$ & $\begin{array}{r}7 \\
125 \\
30\end{array}$ & $\stackrel{3.7}{<}$ & $<$ \\
\hline 41 & Apatite & 9 & 4.7 & 15 & 6200 & 4300 & 35 & 8 & 400000 & 85 & 1.5 & 350 & 50 & 35 & 8 & 30 & $<$ & 9 & 12700 & 15500 \\
\hline $\begin{array}{l}40 \\
39 \\
50\end{array}$ & $\begin{array}{l}\text { Beryl, regular } \\
\text { Beryl, heliodor } \\
\text { Beryl, vorobyevite }\end{array}$ & $\begin{array}{l}42000 \\
41000 \\
39000\end{array}$ & $\begin{array}{l}3 \\
9 \\
1\end{array}$ & $\begin{array}{l}1400 \\
1500 \\
2400\end{array}$ & $\begin{array}{r}17000 \\
9100 \\
6800\end{array}$ & $\begin{array}{r}1200 \\
600 \\
400\end{array}$ & $\begin{array}{r}180 \\
180 \\
90\end{array}$ & $\begin{array}{r}2300 \\
2900 \\
34500\end{array}$ & $\begin{array}{r}500 \\
150 \\
85\end{array}$ & $\underset{2}{<}$ & $\sum_{1}^{<}$ & 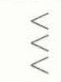 & $\begin{array}{r}100 \\
180 \\
11\end{array}$ & $\begin{array}{l}35 \\
30 \\
60\end{array}$ & $\begin{array}{l}1.0 \\
3.0 \\
2.0\end{array}$ & $\sum_{5}^{<}$ & $\underset{1.0}{<}$ & $\underset{1.0}{<}$ & 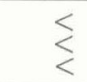 & $\stackrel{<}{<}$ \\
\hline 11 & Columbite & 3 & 3 & 12 & 300 & 500 & 1.0 & 0.8 & 20000 & 20 & 35 & 200 & 100 & 10 & 15 & 30 & 0.9 & 550 & 1000 & 430 \\
\hline 12 & Löllingite & - & - & 7 & 250 & 250 & 8 & 5 & 230 & 5 & $<$ & 8 & 1.3 & 1 & 3.2 & 700000 & 1.0 & 20 & $<$ & $<$ \\
\hline 10 & Cassiterite & 20 & 5 & 120 & 150 & 2500 & 20 & 11 & 500 & 1.7 & 75 & 1.5 & 15 & 10 & 3.0 & 3.0 & 12 & 25 & 13 & 140 \\
\hline 8 & Spodumene & 3.0 & 30 & 16000 & 1600 & 2000 & 15 & 7 & 300 & 13 & - & 10 & 3 & 30 & 10 & 12 & $<$ & $<$ & $<$ & $<$ \\
\hline $\begin{array}{l}23 \\
26\end{array}$ & $\begin{array}{l}\text { Pegmatite } \\
\text { Country rock }\end{array}$ & $\begin{array}{l}6 \\
2\end{array}$ & $\begin{array}{r}1200 \\
3\end{array}$ & $\begin{array}{r}150 \\
30\end{array}$ & $\begin{array}{l}35000 \\
25000\end{array}$ & $\begin{array}{l}45000 \\
37500\end{array}$ & $\begin{array}{l}300 \\
130\end{array}$ & $\begin{array}{r}30 \\
4.0\end{array}$ & $\begin{array}{r}3700 \\
20500\end{array}$ & $\begin{array}{r}30 \\
280\end{array}$ & $\begin{array}{r}30 \\
240\end{array}$ & $\begin{array}{l}10 \\
30\end{array}$ & $\begin{array}{l}30 \\
30\end{array}$ & $\begin{array}{l}25 \\
25\end{array}$ & $\begin{array}{l}2.5 \\
3.0\end{array}$ & $\begin{array}{r}30 \\
3\end{array}$ & $\begin{array}{l}1.5 \\
1.0\end{array}$ & $\begin{array}{l}30 \\
25\end{array}$ & $\begin{array}{r}5 \\
35\end{array}$ & $\begin{array}{r}14 \\
240\end{array}$ \\
\hline & Granite average & 5 & 15 & 30 & 27700 & 33400 & 145 & 5 & 15800 & 285 & 600 & 10 & 40 & 20 & 1.5 & 1.5 & 1.0 & 30 & 40 & 184 \\
\hline
\end{tabular}


Table $5 \mathrm{~b}$. Multielement analyses of Kaatiala minerals and rocks. The contents are given as ppm.

- Not determined < below detection limit

\begin{tabular}{|c|c|c|c|c|c|c|c|c|c|c|c|c|c|c|c|c|c|c|c|}
\hline \multirow[b]{2}{*}{ No. } & \multirow[b]{2}{*}{ Sample } & \multicolumn{4}{|c|}{ Group 5} & \multicolumn{7}{|c|}{ Group 6} & \multicolumn{7}{|c|}{ Group 7} \\
\hline & & $\mathrm{F}$ & $\mathbf{P}$ & $\mathrm{S}$ & $\mathrm{Cl}$ & Sc & V & $\mathrm{Cr}$ & Mn & $\mathrm{Fe}$ & Co & $\mathrm{Ni}$ & $\mathrm{Ti}$ & $\mathrm{Zr}$ & $\mathrm{Nb}$ & Sn & $\mathrm{Ta}$ & Th & $\mathrm{U}$ \\
\hline $\begin{array}{r}28 \\
2 \\
16 \\
33 \\
15\end{array}$ & $\begin{array}{l}\text { Microline perthite } \\
\text { Microcline perthite } \\
\text { Na-rich microcline } \\
\text { Microcline, trans. } \\
\text { Black microcline }\end{array}$ & $\begin{array}{r}215 \\
40 \\
50 \\
1900 \\
450\end{array}$ & $\begin{array}{l}200 \\
175 \\
350 \\
125 \\
500\end{array}$ & $\begin{array}{r}16 \\
15 \\
21 \\
75 \\
480\end{array}$ & $\begin{array}{l}18 \\
55 \\
60 \\
20 \\
60\end{array}$ & $\begin{array}{r}16 \\
15 \\
23 \\
5 \\
12\end{array}$ & $\begin{array}{r}5.3 \\
4 \\
48 \\
4 \\
7\end{array}$ & $\begin{array}{l}49 \\
85 \\
50 \\
55 \\
50\end{array}$ & $\begin{array}{l}19 \\
30 \\
31 \\
15 \\
65\end{array}$ & $\begin{array}{r}340 \\
500 \\
200 \\
800 \\
4700\end{array}$ & $\begin{array}{l}0.1 \\
0.5 \\
0.4 \\
0.6 \\
1.0\end{array}$ & $\begin{array}{r}45 \\
42 \\
23 \\
125 \\
37\end{array}$ & $\begin{array}{l}27 \\
60 \\
33 \\
20 \\
32\end{array}$ & $\begin{array}{l}< \\
1.8 \\
5.6 \\
12\end{array}$ & $\begin{array}{r}1.1 \\
0.2 \\
20 \\
10 \\
4.5\end{array}$ & $\begin{array}{l}3.4 \\
24 \\
7.3 \\
6.5 \\
7.3\end{array}$ & $\begin{array}{r}0.4 \\
\sum \\
5 \\
1.3\end{array}$ & $\begin{array}{l}< \\
\leq \\
7\end{array}$ & $\begin{array}{r}< \\
< \\
7 \\
18\end{array}$ \\
\hline $\begin{array}{l}18 \\
20\end{array}$ & $\begin{array}{l}\text { Oligoclase } \\
\text { Green albite }\end{array}$ & $\begin{array}{r}110 \\
50\end{array}$ & $\begin{array}{l}250 \\
250\end{array}$ & $\begin{array}{r}13 \\
150\end{array}$ & $\begin{array}{l}20 \\
23\end{array}$ & $\begin{array}{l}10 \\
76\end{array}$ & $\begin{array}{l}1.5 \\
1.4\end{array}$ & $\begin{array}{l}17 \\
30\end{array}$ & $\begin{array}{r}4.6 \\
65\end{array}$ & $\begin{array}{r}430 \\
2300\end{array}$ & $\begin{array}{l}0.1 \\
0.4\end{array}$ & $\begin{array}{l}21 \\
13\end{array}$ & $\begin{array}{l}17 \\
18\end{array}$ & 5.8 & $\begin{array}{l}1.3 \\
1.9\end{array}$ & $\overline{3.0}$ & $\stackrel{2.1}{<}$ & $\stackrel{<}{45}$ & < \\
\hline $\begin{array}{r}1 \\
38 \\
37\end{array}$ & $\begin{array}{l}\text { Quartz, regular } \\
\text { Rose quartz } \\
\text { Smoky quartz }\end{array}$ & $\begin{array}{r}17 \\
9 \\
15\end{array}$ & $\begin{array}{l}14 \\
18 \\
12\end{array}$ & $\begin{array}{l}15 \\
12 \\
15\end{array}$ & $\begin{array}{r}11 \\
7.5 \\
5.6\end{array}$ & $\begin{array}{r}1.7 \\
5 \\
5.7\end{array}$ & $\begin{array}{l}0.2 \\
0.4 \\
0.4\end{array}$ & $\begin{array}{l}40 \\
52 \\
59\end{array}$ & $\begin{array}{l}1.6 \\
5.4 \\
6.0\end{array}$ & $\begin{array}{r}280 \\
590 \\
1250\end{array}$ & $\begin{array}{l}0.1 \\
0.1 \\
0.2\end{array}$ & $\begin{array}{l}1.4 \\
9.2 \\
15\end{array}$ & $\frac{17}{58}$ & $\grave{<}$ & $\stackrel{1.5}{<}$ & $\frac{0.6}{-}$ & $\stackrel{4.2}{<}$ & $\underset{<}{25}$ & $\stackrel{<}{<.7}$ \\
\hline $\begin{array}{l}6 \\
7\end{array}$ & $\begin{array}{l}\text { Muscovite } \\
\text { Li-rich mica }\end{array}$ & $\begin{array}{r}8500 \\
22500\end{array}$ & $\begin{array}{l}40 \\
55\end{array}$ & $\begin{array}{r}30 \\
650\end{array}$ & $\begin{array}{l}35 \\
90\end{array}$ & $\begin{array}{l}50 \\
75\end{array}$ & $\begin{array}{l}3.7 \\
6.7\end{array}$ & $\begin{array}{l}20 \\
35\end{array}$ & $\begin{array}{r}550 \\
95\end{array}$ & $\begin{array}{l}2150 \\
1250\end{array}$ & $\begin{array}{l}1.8 \\
1.2\end{array}$ & $\begin{array}{r}14 \\
2.3\end{array}$ & $\begin{array}{r}1000 \\
820\end{array}$ & $\begin{array}{l}6.2 \\
3.3\end{array}$ & $\begin{array}{l}150 \\
250\end{array}$ & $\begin{array}{l}160 \\
220\end{array}$ & $\begin{array}{l}30 \\
70\end{array}$ & $<$ & $\stackrel{1.1}{<}$ \\
\hline $\begin{array}{r}4 \\
34 \\
35\end{array}$ & $\begin{array}{l}\text { Tourmaline, schorl. } \\
\text { Green tourmaline } \\
\text { Red tourmaline }\end{array}$ & $\begin{array}{r}3900 \\
25000 \\
7500\end{array}$ & $\begin{array}{l}40 \\
26 \\
67\end{array}$ & $\begin{array}{l}45 \\
41 \\
24\end{array}$ & $\begin{array}{l}42 \\
16 \\
20\end{array}$ & $\begin{array}{r}13 \\
2 \\
1\end{array}$ & $\begin{array}{r}37 \\
13 \\
8\end{array}$ & $\begin{array}{l}69 \\
64 \\
61\end{array}$ & $\begin{array}{l}2500 \\
5000 \\
3600\end{array}$ & $\begin{array}{r}58700 \\
32000 \\
4600\end{array}$ & $\begin{array}{r}0.2 \\
10 \\
8\end{array}$ & $\begin{array}{l}60 \\
80 \\
50\end{array}$ & $\begin{array}{l}620 \\
100 \\
400\end{array}$ & $\begin{array}{l}10 \\
10 \\
15\end{array}$ & $\begin{array}{r}40 \\
40 \\
4.9\end{array}$ & $\begin{array}{r}19 \\
120 \\
100\end{array}$ & $\begin{array}{l}1.4 \\
5.3 \\
75\end{array}$ & $\begin{array}{l}1.8 \\
\underset{5.9}{<}\end{array}$ & $\stackrel{1.4}{<}$ \\
\hline 41 & Apatite & 12500 & - & 55 & 36 & - & 3.7 & 7.5 & 6200 & 1260 & $<$ & 2500 & 100 & 20 & - & 11 & - & 49 & 12 \\
\hline $\begin{array}{l}40 \\
39 \\
50\end{array}$ & $\begin{array}{l}\text { Beryl, regular } \\
\text { Beryl, heliodor } \\
\text { Beryl, vorobyevite }\end{array}$ & $\begin{array}{r}42 \\
7 \\
-\end{array}$ & $\begin{array}{r}15 \\
1.2 \\
110\end{array}$ & $\begin{array}{l}34 \\
16 \\
-\end{array}$ & $\begin{array}{l}14 \\
14 \\
-\end{array}$ & $\begin{array}{r}4 \\
12 \\
25\end{array}$ & $\frac{1.5}{1}$ & $\begin{array}{r}86 \\
160 \\
85\end{array}$ & $\begin{array}{r}30 \\
160 \\
50\end{array}$ & $\begin{array}{r}1800 \\
800 \\
660\end{array}$ & 二 & $\stackrel{2}{<}$ & $\begin{array}{l}25 \\
40 \\
25\end{array}$ & $\bar{Z}$ & $\frac{47}{-}$ & $\begin{array}{r}2 \\
2 \\
45\end{array}$ & 二 & 二 & Z \\
\hline 11 & Columbite & 90 & 18 & 175 & 52 & 420 & 0.7 & 1.1 & 47000 & 150000 & 9 & 75 & 16700 & & 500000 & 1700 & 41000 & 24 & 1300 \\
\hline 12 & Löllingite & 150 & 7.5 & 5000 & 15 & 0.2 & 1.2 & 1.6 & 10 & 200000 & 4.6 & 42 & 30 & 1.8 & 4.6 & 42 & 70 & $<$ & $<$ \\
\hline 10 & Cassiterite & 12 & 75 & 19 & 11 & 8.7 & 1.5 & 1.1 & 1500 & 9300 & 930 & 25000 & 2050 & 1350 & - & - & - & $<$ & 3.8 \\
\hline 8 & Spodumene & 120 & 20 & 12 & 30 & - & 13 & 30 & 720 & 8300 & 1.2 & 2.3 & 40 & 0.4 & 1.5 & 110 & 8.3 & $<$ & $<$ \\
\hline $\begin{array}{l}23 \\
26\end{array}$ & $\begin{array}{l}\text { Pegmatite } \\
\text { Country rock }\end{array}$ & $\begin{array}{r}1000 \\
500\end{array}$ & $\begin{array}{l}250 \\
200\end{array}$ & $\begin{array}{l}30 \\
50\end{array}$ & $\begin{array}{l}30 \\
30\end{array}$ & $\begin{array}{r}4 \\
10\end{array}$ & $\begin{array}{r}4 \\
50\end{array}$ & $\begin{array}{l}40 \\
50\end{array}$ & $\begin{array}{r}30 \\
300\end{array}$ & $\begin{array}{r}7000 \\
30000\end{array}$ & $\begin{array}{r}5 \\
20\end{array}$ & $\begin{array}{r}30 \\
7\end{array}$ & $\begin{array}{r}500 \\
3000\end{array}$ & $\begin{array}{l}13 \\
40\end{array}$ & $\begin{array}{l}60 \\
23\end{array}$ & $\begin{array}{r}12 \\
1.5\end{array}$ & $\begin{array}{l}4.3 \\
1.2\end{array}$ & $\begin{array}{r}3.2 \\
6\end{array}$ & $\begin{array}{l}3.3 \\
2.3\end{array}$ \\
\hline & Granite average & 850 & 700 & 270 & 200 & 6 & 40 & 10 & 400 & 27000 & 2 & 4 & 2300 & 180 & 20 & 3 & 3.5 & 17 & 4.8 \\
\hline
\end{tabular}


Table 6. Bulk composition of the Kaatiala pegmatite.

\begin{tabular}{|c|c|c|c|c|}
\hline & $\begin{array}{l}\text { Main components } \\
\text { of bulk sample } \\
\% \text { by weight }\end{array}$ & $\begin{array}{l}\text { Tourmaline and } \\
\text { micas deducted } \\
\% \% \text { by weight }\end{array}$ & \multicolumn{2}{|c|}{$\begin{array}{l}\text { Calculated mode } \\
\text { for bulk samplec }\end{array}$} \\
\hline $\mathrm{SiO}_{2}$ & 71.41 & 73.71 & Quartz & 212 \\
\hline $\mathrm{Al}_{2} \mathrm{O}_{3}$ & 15.41 & 14.68 & Microcline & 35.6 \\
\hline $\mathrm{Fe}_{2} \mathrm{O}_{3}$ & 0.81 & 0.17 & Albite & 32.5 \\
\hline $\mathrm{MgO}$ & 0.28 & 0.09 & Micas & 6.0 \\
\hline $\mathrm{CaO}$ & 0.52 & 0.52 & Tourmaline & 4.0 \\
\hline $\mathrm{Na}_{2} \mathrm{O}$ & 4.60 & 4.91 & Others & 0.7 \\
\hline $\begin{array}{l}\mathrm{K}_{2} \mathrm{O} \\
\text { Rest }\end{array}$ & $\begin{array}{l}5.40 \\
1.24\end{array}$ & 5.33 & & 100.0 \\
\hline \multirow{2}{*}{ Rest } & $\begin{array}{l}\text { Trace elements forming } \\
\text { own specific mineral }\end{array}$ & $\begin{array}{l}\text { Disperced in the } \\
\text { other minerals }\end{array}$ & \multicolumn{2}{|c|}{$\begin{array}{c}\text { Modal composition } \\
\text { of specific } \\
\text { minerals }\end{array}$} \\
\hline & $\mathrm{ppm}$ & $\%$ & & $\%$ \\
\hline $\mathrm{Be}$ & 6 & 40 & Beryl & 0.01 \\
\hline B & 1200 & 0.5 & Tourmaline & 4.0 \\
\hline $\mathrm{P}$ & 250 & 50 & Apatite & 0.06 \\
\hline As & 30 & 5 & Löllingite & 0.004 \\
\hline $\mathrm{Nb}$ & 35 & 30 & Columbite & 0.005 \\
\hline
\end{tabular}

a Analyst: v. Weymarn, P-O, Partek Oy.

b Approximative chemical analyses for micas and tourmaline in weight per cent: $\mathrm{SiO}_{2} 62$ and 42 , $\mathrm{Al}_{2} \mathrm{O}_{3} 20$ and 25, $\mathrm{Fe}_{2} \mathrm{O}_{3} 1$ and $15, \mathrm{MgO} 2$ and 2, $\mathrm{CaO} 0.5$ and 0.5 , $\mathrm{Na}_{2} \mathrm{O} 1$ and 3 , and $\mathrm{K}_{2} \mathrm{O} 10$ and 0 .

c Quartz $=\mathrm{SiO}_{2}$, Microcline $\mathrm{Or}_{80} \mathrm{Ab}_{20}$, Albite $\mathrm{Ab}_{94} \mathrm{An}_{6}$, Micas: approx. 75 per cent muscovite +25 per cent lithia mica.

d Estimates base on the mode of the main minerals and their typical analyses.

amounts of micas were obtained from the flotation tests, and the amount of tourmaline from the boron content (1200 ppm) of the bulk sample. Oxides bound in micas and tourmaline were deducted from the bulk chemical analysis, and the amounts of other minerals were calculated from the residual. Owing to the varying chemical compositions of both tourmaline and micas, the deduction was based on approximate chemical analyses.

The content of micas ( $6 \mathrm{wt-} \%$ ) corresponds well to the values of zoned pegmatites, e.g. the Hugo pegmatite, Keystone, USA, whose weighted mica content is also 6 per cent (Norton et al., 1962). In unzoned pegmatites, on the other hand, the mica content is mostly below 4 per cent (Norton 1970).
Like the percentage of tourmaline, the percentages of beryl, apatite, löllingite and columbite were calculated from the contents of $\mathrm{Be}, \mathrm{P}, \mathrm{As}$, and $\mathrm{Nb}$ in the bulk sample.

Tin and lithium are dispersed to such a high degree, especially in micas, that it was not possible with the aid of trace element analyses to calculate the amounts of their minerals proper, cassiterite and spodumene. According to mineralogical observations made during the operational period, their quantities are without significance and have no influence on chemical balance.

Table 7 gives the total chemical and mineralogical balance of the whole Kaatiala pegmatite, estimated on the basis of the amounts of minerals and their chemical analyses. For 
Table 7. The chemical and mineralogical balance of the Kaatiala pegmatite

\begin{tabular}{|c|c|c|c|c|c|c|c|c|c|c|c|}
\hline \multicolumn{2}{|c|}{$\begin{array}{l}\text { Mineral } \\
\text { Percent of total } \\
\text { Quantity, tons }\end{array}$} & \multirow{2}{*}{ 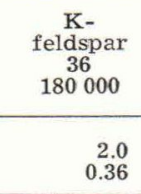 } & \multirow{2}{*}{$\begin{array}{c}\text { Na- } \\
\text { feldspar } \\
33 \\
165000 \\
\\
1.0 \\
0.17\end{array}$} & \multirow{2}{*}{ 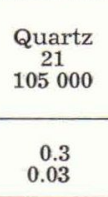 } & \multirow{2}{*}{$\begin{array}{c}\begin{array}{c}\text { Mica } \\
6 \\
30000\end{array} \\
\begin{array}{c}10-50 \\
0.3-1.5\end{array}\end{array}$} & \multirow{2}{*}{$\begin{array}{c}\begin{array}{c}\text { Tour- } \\
\text { maline } \\
4 \\
20000\end{array} \\
2.0 \\
0.04\end{array}$} & \multirow{2}{*}{$\begin{array}{c}\text { Apatite } \\
0.06 \\
300 \\
\\
\\
\end{array}$} & \multirow{2}{*}{$\begin{array}{c}\text { Beryl } \\
0.01 \\
50 \\
\\
36000 \\
1.8\end{array}$} & \multirow{2}{*}{$\begin{array}{c}\text { Colum } \\
\text { bite } \\
0.005 \\
25 \\
\\
= \\
=\end{array}$} & \multirow{2}{*}{$\begin{array}{c}\text { Löll- } \\
\text { ingite } \\
0.004 \\
20 \\
= \\
=\end{array}$} & \multirow{2}{*}{ 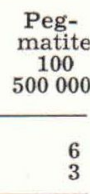 } \\
\hline $\mathrm{Be}$ & $\begin{array}{l}\text { ppm } \\
\text { tons }\end{array}$ & & & & & & & & & & \\
\hline B & $\begin{array}{l}\text { ppm } \\
\text { tons }\end{array}$ & $\begin{array}{r}5 \\
0.9\end{array}$ & $\begin{array}{r}0.7 \\
0.12\end{array}$ & $\begin{array}{r}3 \\
0.3\end{array}$ & $\begin{array}{r}70 \\
2.1\end{array}$ & $\begin{array}{r}30000 \\
600\end{array}$ & $\underline{5}$ & $\underline{3}$ & $\underline{3}$ & 二 & $\begin{array}{r}1200 \\
600\end{array}$ \\
\hline $\mathrm{Li}$ & $\begin{array}{l}\text { ppm } \\
\text { tons }\end{array}$ & $\begin{array}{r}50 \\
9.0\end{array}$ & $\begin{array}{r}2 \\
0.3\end{array}$ & $\begin{array}{r}5 \\
0.5\end{array}$ & $\begin{array}{r}1500-7000 \\
45-210\end{array}$ & $\begin{array}{r}50 \\
1\end{array}$ & 15 & $\begin{array}{r}1400 \\
0.1\end{array}$ & 12 & 7 & $\begin{array}{r}150 \\
75\end{array}$ \\
\hline $\mathrm{Rb}$ & $\begin{array}{l}\text { ppm } \\
\text { tons }\end{array}$ & $\begin{array}{r}300-800 \\
54-144\end{array}$ & $\begin{array}{r}2 \\
0.3\end{array}$ & $\begin{array}{r}0.2 \\
0.02\end{array}$ & $\begin{array}{r}1500-3000 \\
45-90\end{array}$ & $\begin{array}{r}5 \\
0.1\end{array}$ & 35 & $\begin{array}{l}180 \\
0.01\end{array}$ & 1 & $\frac{8}{-}$ & $\begin{array}{l}300 \\
150\end{array}$ \\
\hline Cs & $\begin{array}{l}\text { ppm } \\
\text { tons }\end{array}$ & $\begin{array}{r}20-35 \\
3.6-6.3\end{array}$ & $\begin{array}{l}0.5 \\
0.1\end{array}$ & $\frac{0.2}{-}$ & $\begin{array}{r}120-2200 \\
3.6-66\end{array}$ & 0.2 & $\frac{7.5}{-}$ & $\begin{array}{r}2300 \\
0.1\end{array}$ & 0.8 & - & $\begin{array}{l}30 \\
15\end{array}$ \\
\hline $\mathrm{Ca}$ & $\begin{array}{l}\text { ppm } \\
\text { tons }\end{array}$ & $\begin{array}{r}1500-2000 \\
270-360\end{array}$ & $\begin{array}{l}6700 \\
1140\end{array}$ & $\begin{array}{r}110 \\
11\end{array}$ & $\begin{array}{r}1000 \\
30\end{array}$ & $\begin{array}{r}950 \\
19\end{array}$ & $\begin{array}{r}400000 \\
120\end{array}$ & 500 & $\begin{array}{r}20000 \\
0.5\end{array}$ & 230 & $\begin{array}{l}3700 \\
1850\end{array}$ \\
\hline $\mathrm{Sr}$ & $\begin{array}{l}\text { ppm } \\
\text { tons }\end{array}$ & $\begin{array}{c}9-50 \\
1.6-9\end{array}$ & $\begin{array}{r}45 \\
7.4\end{array}$ & $\begin{array}{r}1 \\
0.1\end{array}$ & $\begin{array}{l}11 \\
0.1\end{array}$ & $\frac{2.5}{-}$ & 85 & 二 & 20 & 5 & $\begin{array}{l}30 \\
15\end{array}$ \\
\hline $\mathrm{Ba}$ & $\begin{array}{l}\text { ppm } \\
\text { tons }\end{array}$ & $\begin{array}{r}10-20 \\
1.8-21.6\end{array}$ & 二 & $=$ & 1.5 & 二 & 15 & 二 & 35 & - & $\begin{array}{l}30 \\
15\end{array}$ \\
\hline $\mathrm{Ga}$ & $\begin{array}{l}\text { ppm } \\
\text { tons }\end{array}$ & $\begin{array}{r}15-20 \\
2.7-3.6 \\
\end{array}$ & $\begin{array}{r}25 \\
4.1\end{array}$ & $=$ & $\begin{array}{r}65-100 \\
2-3 \\
\end{array}$ & $\begin{array}{l}130 \\
2.6\end{array}$ & $\frac{35}{-}$ & $\frac{35}{-}$ & 10 & 1 & $\begin{array}{r}25 \\
12.5\end{array}$ \\
\hline $\mathrm{Ge}$ & $\begin{array}{l}\text { ppm } \\
\text { tons }\end{array}$ & $\begin{array}{r}1 \\
0.18\end{array}$ & $\begin{array}{r}4 \\
0.66\end{array}$ & $\begin{array}{r}1.7 \\
0.17\end{array}$ & $0.15-0.30$ & $\begin{array}{r}3.4 \\
0.06\end{array}$ & 8 & 1 & 15 & $\begin{array}{l}3.2 \\
--\end{array}$ & $\begin{array}{l}2.5 \\
1.3\end{array}$ \\
\hline As & $\begin{array}{l}\text { ppm } \\
\text { tons }\end{array}$ & $\begin{array}{r}1.0 \\
0.18\end{array}$ & $\begin{array}{r}1.8 \\
0.29\end{array}$ & $\begin{array}{r}0.1 \\
0.10\end{array}$ & $\begin{array}{r}10-15 \\
0.30-0.45\end{array}$ & $\begin{array}{r}65 \\
1.3\end{array}$ & 30 & 二 & 30 & $\begin{array}{r}700000 \\
14\end{array}$ & $\begin{array}{l}30 \\
15\end{array}$ \\
\hline $\mathrm{TI}$ & $\begin{array}{l}\text { ppm } \\
\text { tons }\end{array}$ & $\begin{array}{r}2.2-5 \\
0.40-0.90\end{array}$ & $=$ & 二 & $0.15-0.45$ & 二 & 二 & 二 & $\underline{0.9}$ & 1 & $\begin{array}{r}1.5 \\
0.75\end{array}$ \\
\hline $\mathrm{Pb}$ & $\begin{array}{l}\text { ppm } \\
\text { tons }\end{array}$ & $\begin{array}{r}50-100 \\
9-18\end{array}$ & $\begin{array}{r}11 \\
1.8\end{array}$ & 二 & $\begin{array}{r}6 \\
0.18\end{array}$ & $\begin{array}{r}7 \\
0.14\end{array}$ & $\frac{9}{-}$ & - & $\begin{array}{r}550 \\
0.03\end{array}$ & 20 & $\begin{array}{l}30 \\
15\end{array}$ \\
\hline F & $\begin{array}{l}\text { ppm } \\
\text { tons }\end{array}$ & $\begin{array}{r}40-215 \\
7.2-38.7 \\
\end{array}$ & $\begin{array}{r}110 \\
18.0\end{array}$ & $\begin{array}{r}17 \\
1.7 \\
\end{array}$ & $\begin{array}{r}8500-22500 \\
255-675\end{array}$ & $\begin{array}{r}3900 \\
68 \\
\end{array}$ & $\begin{array}{r}12500 \\
3.8\end{array}$ & $\frac{42}{-}$ & $\frac{90}{-}$ & 150 & $\begin{array}{r}1000 \\
500\end{array}$ \\
\hline $\mathrm{P}$ & $\begin{array}{l}\text { ppm } \\
\text { tons }\end{array}$ & $\begin{array}{r}175-200 \\
31.5-36.0\end{array}$ & $\begin{array}{r}250 \\
41.3\end{array}$ & $\begin{array}{r}14 \\
1.4 \\
\end{array}$ & $\begin{array}{r}40-55 \\
1.2-1.7\end{array}$ & $\begin{array}{r}40 \\
0.8\end{array}$ & $\begin{array}{r}180000 \\
54.0\end{array}$ & 15 & 18 & 7.5 & $\begin{array}{l}250 \\
125\end{array}$ \\
\hline $\mathrm{Nb}$ & $\begin{array}{l}\text { ppm } \\
\text { tons }\end{array}$ & $\begin{array}{r}0.2-1.1 \\
0.04-0.2\end{array}$ & $\begin{array}{l}1.3 \\
0.2\end{array}$ & $\begin{array}{r}1.5 \\
0.15\end{array}$ & $\begin{array}{r}150-250 \\
4.5-7.5\end{array}$ & $\begin{array}{l}4.0 \\
0.1\end{array}$ & - & 4.7 & $\begin{array}{r}500000 \\
12.5 \\
\end{array}$ & $\stackrel{4.6}{-}$ & $\begin{array}{r}35 \\
17.5\end{array}$ \\
\hline Sn & $\begin{array}{l}\text { ppm } \\
\text { tons }\end{array}$ & $\begin{array}{l}2.4-3.4 \\
0.4-0.6\end{array}$ & 二 & $\begin{array}{l}0.6 \\
0.1\end{array}$ & $\begin{array}{r}160-220 \\
4.8-6.6\end{array}$ & $\begin{array}{l}19 \\
0.3\end{array}$ & 11 & $\frac{2}{-}$ & 1700 & $\frac{42}{-}$ & $\begin{array}{r}12 \\
6\end{array}$ \\
\hline $\mathrm{Ta}$ & $\begin{array}{l}\text { ppm } \\
\text { tons }\end{array}$ & $\begin{array}{r}0.4 \\
0.07\end{array}$ & $\begin{array}{r}2.1 \\
0.35\end{array}$ & $\begin{array}{r}4.2 \\
0.42\end{array}$ & $\begin{array}{r}30-70 \\
0.9-2.1\end{array}$ & 1.4 & Z & - & $\begin{array}{r}41000 \\
1.03\end{array}$ & $\frac{6.7}{-}$ & $\begin{array}{r}4.3 \\
2.15\end{array}$ \\
\hline Th & $\begin{array}{l}\text { ppm } \\
\text { tons }\end{array}$ & $\begin{array}{r}0-7 \\
0-1.26\end{array}$ & $\begin{array}{r}0-45 \\
0-7.4\end{array}$ & 二 & 二 & $\begin{array}{r}1.8 \\
0.03\end{array}$ & $\begin{array}{r}49 \\
0.01\end{array}$ & 二 & 24 & 二 & $\begin{array}{r}3.2 \\
1.60 \\
\end{array}$ \\
\hline U & $\begin{array}{l}\text { ppm } \\
\text { tons }\end{array}$ & $\begin{array}{r}0-18 \\
0-3.2 \\
\end{array}$ & $\begin{array}{c}0-20 \\
0-3.3 \\
\end{array}$ & - & $\begin{array}{r}1.1-2.8 \\
0.03-0.08\end{array}$ & $\begin{array}{r}1.4 \\
0.02\end{array}$ & 12 & 二 & $\begin{array}{r}1300 \\
0.03 \\
\end{array}$ & 二 & $\begin{array}{r}3.3 \\
1.65 \\
\end{array}$ \\
\hline REE & $\begin{array}{l}\text { ppm } \\
\text { tons }\end{array}$ & $\begin{array}{r}0-14 \\
0-2.52\end{array}$ & $\begin{array}{r}0-11 \\
0-1.85\end{array}$ & - & 二 & 二 & $\begin{array}{r}15500 \\
4.65\end{array}$ & 二 & $\begin{array}{l}430 \\
0.01\end{array}$ & $=$ & $\begin{array}{r}13 \\
6.50\end{array}$ \\
\hline
\end{tabular}

convenience, the total amount of the quarried pegmatite is rounded from 516000 to 500000 tons.

\section{Discussion}

The analytical data are presented in the manner proposed by Taylor (1965), this being a convenient way of arranging trace element data for many elements. This arrangement is based largely on geochemical association.

Within each group of elements of similar geochemical behaviour, the arrangement is not in the order of decreasing radius as Taylor has advised but in the order of atomic 
numbers. The values for the granite average proposed by Taylor (1964) are used for comparison. The enrichment factor is the ratio of the content of any element in the pegmatite body to that of the granite average. The concentration coefficients for an element were calculated according to the equation $\frac{\mathrm{k} \cdot 100}{\mathrm{k}+\mathrm{p}}$, where $\mathrm{k}$ is the amount of the element in its minerals proper and $\mathrm{p}$ is the amount of the disseminated element in the pegmatite except in its own minerals (Beus 1966).

\section{The small cations Be and $B$.}

\section{Table 5a, group 1}

The beryllium enrichment factor is only 1.2. Despite the low beryllium content, beryl was formed, although its abundance is only 0.01 $\%$. The coefficient of beryllium concentration is $60 \%$. The highest contents of disseminated beryllium are in lithium-rich mica, $50 \mathrm{ppm}$ and in coloured tourmalines, $11-15 \mathrm{ppm}$. The relatively high degree of dissemination is typical of granitic pegmatites containing rare metals and small amounts of beryllium (Beus 1966).

The enrichment factor for boron in the Kaatiala pegmatite is 80 . This is the highest enrichment factor of any element in the pegmatite determined in this work. The country rock, on the other hand, shows a negative boron anomaly, i.e. only $6 \mathrm{ppm}$. The granite average for boron is $15 \mathrm{ppm}$.

Boron is concentrated in tourmaline, the coefficient of concentration exeeding 99 per cent. The content of boron in all the other minerals except micas is remarkably low, that is, below $10 \mathrm{ppm}$.

According to Oftedal (1964), pegmatite minerals containing rare earths are often relatively rich in boron, up to several tenths of a per cent; nevertheless, columbite and apatite, the only rare earth element carriers, contain only 4.7 and 3.0 ppm B, respectively.
The mobile complexes, in which form boron is transported, are probably borontrihalides or even $\mathrm{CsBF}_{4}$ as proposed by Stavorov et al. (1962). The relatively high content of fluorine and cesium in the pegmatite corroborates this theory.

Bräuer (1970), too, points to the marked influence of fluorine on the formation of tourmaline. He suggests that the $\mathrm{BF}_{4}{ }^{-}$complex gets concentrated into the last granitic melts and forms, during the progress of autometasomatism, independent tourmaline crystals.

The large cations including alkalies $\mathrm{Li}, \mathrm{Na}$, $\mathrm{K}, \mathrm{Rb}$, and $\mathrm{Cs}$ and alkaline earths $\mathrm{Ca}, \mathrm{Sr}$ and Ba. Table 5 a, group 2

All these elements, except $\mathrm{Li}$, occur in eight to twelvefold coordination with oxygen in silicates. Lithium generally occurs in sixfold coordination, isomorphously replacing $\mathrm{Mg}, \mathrm{Fe}^{2+}$ and Al. They all form dominantly ionic bonds.

The alkalies are all enriched in the pegmatite body compared with the granite average. The enrichment factor for lithium is 5.0, for sodium 1.2, for potassium 1.3, for rubidium 2.1 and for cesium 6.0.

If all micas are regarded as Li-bearing minerals, the coefficient of lithium concentration is 82 . The proper Li-mineral, spodumene, is so rare, that its Li-content is without significance in this calculation. Except in Lispecific minerals, the Li-content varies as follows: Vorobyevite (Cs-rich beryl) contains 2400 ppm lithium, other beryl varieties 1500 $-1400 \mathrm{ppm}$, coloured tourmaline 800-1000 ppm, black tourmaline $50 \mathrm{ppm}$, rose and smoky quartz 35-40 ppm, normal quartz 5 ppm, K-feldspars from $20 \mathrm{ppm}$ to $700 \mathrm{ppm}$ in the last crystallization forms and $\mathrm{Na}$ feldspars from $2.1 \mathrm{ppm}$ to $14 \mathrm{ppm}$.

Sodium and potassium do not show any 
marked tendency to enrichment except in their minerals proper, $\mathrm{Na}$ - and $\mathrm{K}$-feldspars and micas. Normal quartz contained about 5 ppm $\mathrm{Na}$ and $40 \mathrm{ppm} \mathrm{K}$. The tenors in the coloured varieties were slightly higher.

Tourmalines are all relatively poor in $\mathrm{K}$, from $140 \mathrm{ppm}$ to $2300 \mathrm{ppm}$, but richer in $\mathrm{Na}$, $12000-21000 \mathrm{ppm}$. No big differences were observed in the $\mathrm{K}+\mathrm{Na}$ contents between coloured and black tourmalines. Spodumene is relatively poor in both $\mathrm{Na}, 1600 \mathrm{ppm}$ and $\mathrm{K}, 2000 \mathrm{ppm}$.

The enrichment factor for $\mathrm{Rb}, 2.1$, is too low, especially regarding the marked abundance of $\mathrm{K}$ in pegmatite, to form its own minerals; it is, therefore, completely dispersed in K-feldspar and micas (Povarennykh 1972). All the other minerals are very poor in Rb, mostly below $25 \mathrm{ppm}$, except beryls with $90-180 \mathrm{ppm}$.

The cesium content of the whole pegmatite is $30 \mathrm{ppm}$. It has been concentrated in the cesium beryl vorobyevite, which contained 34500 ppm Cs.

Lithium-rich mica is another mineral with high cesium content, $2000 \mathrm{ppm}$. The tenor of cesium in muscovite is much lower, only 120 ppm. Like rubidium, the bulk of the cesium is dispersed in $\mathrm{K}$-feldspar, which normally contains $20-50 \mathrm{ppm}$ Cs. Nevertheless, the transparent K-feldspar variety found in the cavity representing the last stage of crystallization contained as much as $800 \mathrm{ppm} \mathrm{Cs}$ and 2500 ppm Rb. Lappalainen and Neuvonen (1968) found at Kaatiala a specimen of Kfeldspar which contained $490 \mathrm{ppm} \mathrm{Cs}$ and 9400 ppm Rb.

Unlike the alkalies, the alkaline earths show a clear deficiency compared with the granite average, calcium 0.23 , strontium 0.11 and barium 0.05. Calcium occurs in its own minerals, plagioclase and apatite. These minerals also contain most of the dispersed strontium. Barium, on the other hand, is dispersed into $\mathrm{K}$-feldspar, the highest content being $120 \mathrm{ppm}$ in the early crystallization forms.

\section{The chalcophile elements $\mathrm{Cu}, \mathrm{Zn}, \mathrm{Ga}, \mathrm{Ge}, \mathrm{As}$,} Tl, and $\mathbf{P b}$. Table 5 a group 3

Copper is concentrated into apatite and columbite, and zinc and gallium into tourmalines. Germanium is almost ubiquitous in all minerals, and shows a low degree of concentration in micas, columbite and laststage K-feldspar.

The contents of $\mathrm{Cu}, \mathrm{Zn}, \mathrm{Ga}$ and $\mathrm{Ge}$ are almost identical with the average granitic values of these elements. The enrichment factor of arsenic compared with the granite average is 20. It forms its own mineral, löllingite, which contains nearly 95 per cent of the total arsenic.

Thallium is mainly concentrated into laststage $\mathrm{K}$-feldspar and lithia mica, whereas lead is most abundant in early stage K-feldspar, columbite and green tourmaline.

\section{The rare earth elements La-Lu and Y. Table $5 c$, group 4}

The rare earth elements in Kaatiala pegmatite show the highest contents in apatite, which assays $15500 \mathrm{ppm}$ lanthanoids and 12700 ppm Y. Columbite is another concentrator of rare earths although the abundances in it are clearly lower, only $430 \mathrm{ppm}$ lanthanoids and $1000 \mathrm{ppm}$ Y. Together these minerals contain about 70 per cent of the rare earth elements in the whole pegmatite. The remaining 30 per cent are to be found in feldspars.

The mean content of lanthanoids in the Kaatiala pegmatite is $14 \mathrm{ppm}$ and that of yttrium 5 ppm. These contents are much lower, only 7.5 and 12.5 per cent, respective, of those of the same elements in the average 


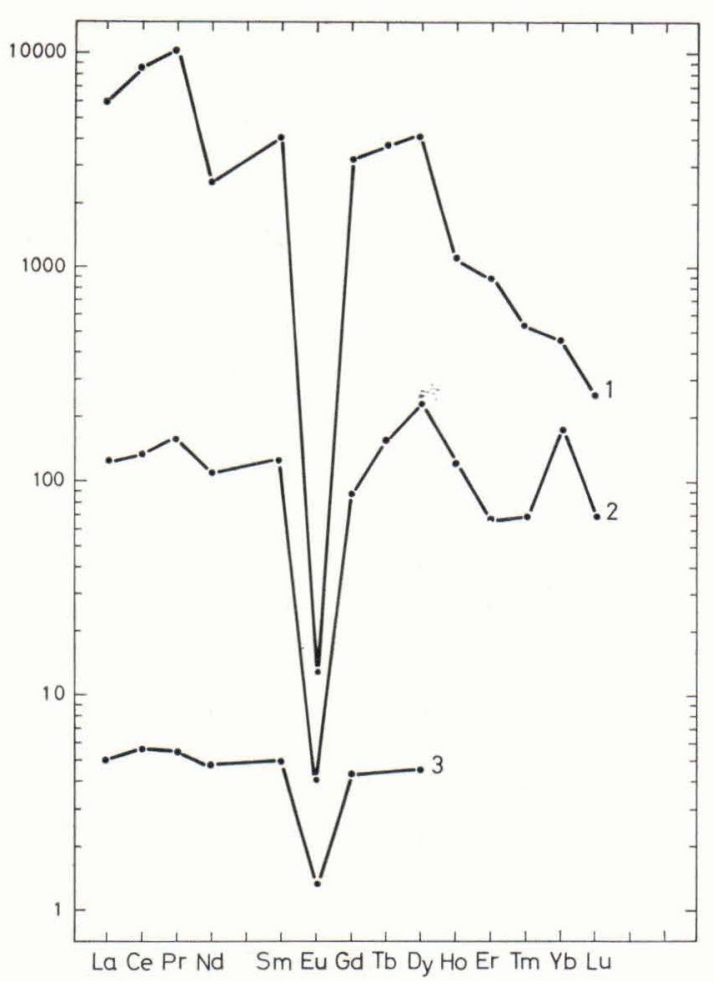

Fig. 4. Distribution patterns of the lanthanoids in Kaatiala apatite (1), columbite (2) and the total pegmatite (3) normalized against chondrite.

granite, which are $184 \mathrm{ppm}$ for lanthanoids and $40 \mathrm{ppm}$ for yttrium (Taylor 1968).

The distribution patterns of the lanthanoids in Kaatiala apatite, columbite, and the whole pegmatite normalized against chondrite, (Nance et al. 1976) are shown in Fig. 4. Typical of all are the pronounced deficiency of europium and neodymium, and the elevated value of dysprosium. Columbite also shows an elevated value for ytterbium, which is consistent with its high yttrium content.

\section{The anions of $\mathbf{F}, \mathbf{P}, \mathbf{S}$ and $\mathbf{C l}$. Table $5 \mathrm{~b}$, group 5}

Kaatiala pegmatite averages 1000 ppm fluorine. Most of the fluorine, about $80 \%$, is found in micas. In these minerals fluorine ion substitutes for hydroxyl. The content of fluorine is lowest in all varieties of quartz, 9-17 ppm; in other minerals it is as follows: $7-42 \mathrm{ppm}$ in beryls, $40-1900 \mathrm{ppm}$ in $\mathrm{K}-$ feldspar, 50-110 ppm in Na-feldspar, 3900$25000 \mathrm{ppm}$ in tourmalines and $12500 \mathrm{ppm}$ in apatite.

The average content of phosphorus in the pegmatite is $250 \mathrm{ppm}$, which is about one third of that of the average granite. Nevertheless, despite this low value, small amounts of apatite have formed, mostly on the border zone. Apatite contains about 50 per cent of the total phosphorus. The remaining 50 per cent is mainly in $\mathrm{Na}$ - and $\mathrm{K}$-feldspars.

The average content of sulphur in the whole pegmatite is only $30 \mathrm{ppm}$, which is one ninth of the granite average. In addition to löllingite, only lithium-rich mica, biotite and columbite concentrate sulphur to some degree.

In spite of relatively high fluorine tenor, the Kaatiala pegmatite is poor in chlorine. The average content is $30 \mathrm{ppm}$, which is only one sixth of that in the average granite. Chlorine exhibits almost constant abundances in all minerals, ranging from $5.6-60 \mathrm{ppm}$ in quartz and feldspars to $90 \mathrm{ppm}$ in lithium-rich mica. Even in apatite the chlorine content is as low as $36 \mathrm{ppm}$.

The trivalent and divalent cations Sc, V, Cr, Mn, Fe, Co and Ni. Table 5 b, group 6

Scandium is an ubiquitous element in all the Kaatiala minerals. The only significant scandium carrier, however, is columbite, which contains 420 ppm scandium. Haapala et. al. (1967) have found that a variety of columbite of Haapaluoma pegmatite, about 16 $\mathrm{km}$ southwest of Kaatiala, contained 0.90 per cent $\mathrm{Sc}_{2} \mathrm{O}_{3}$.

Owing to the small amount of columbite and its low scandium content, the overall 
scandium tenor in Kaatiala pegmatite is 4 ppm, which is slightly less than in the average granite, $6 \mathrm{ppm}$.

Vanadinium is concentrated into black tourmaline. Its mean content is 10 per cent of the granite average.

Chromium is a disperse element without any significant accumulation tendency. Its enrichment factor is 4 in the whole pegmatite.

Manganese shows the highest concentration in columbite, iron in löllingite and columbite, cobalt and nickel in cassiterite. The manganese and iron contents are distinctly lower and the abundances of cobalt and nickel higher than those of the granite averages.

It is of great practical importance that all these elements occur in negligible amounts in feldspars and quartz. They all have a colouririg effect on silicate melts; thus, elevated abundances of these elements are harmful in ceramic raw materials.

\section{The large highly charged cations with valencies 4,5 or 6 including $\mathrm{Ti}, \mathrm{Zr}, \mathrm{Nb}, \mathrm{Sn}$, Ta, Th and U. Table $5 \mathrm{~b}$, group 7}

These elements do not readily enter common rock-forming minerals owing to valency difficulties. They therefore occur in the last hydrous fluids that migrate through the fractures formed after consolidation of the main pegmatite body. Albite, micas, spodumene, cassiterite, coloured tourmalines, columbite and löllingite crystallize from these fluids directly or through hydrothermal processes. Thus, these minerals are the main carriers of elements of this subgroup.

Columbite and micas contain over 95 per cent of the total of niobium and tantalum. Cassiterite is the main concentrator of tin and zirconium, although the bulk of the tin is found in micas. Löllingite is the only concentrator of arsenic and columbite that of titanium.

The zirconium content in the pegmatite is far below, and the niobium, tin and tantalum contents distinctly higher than in the average granite.

The mean contents of thorium and uranium are also below the granite average. Thorium is concentrated in apatite, and uranium in columbite. The bulk of the thorium and uranium are, however, in feldspars of the fracture and replacement zones. Uraniumrich black microcline contained $18 \mathrm{ppm} \mathrm{U}$ and $7 \mathrm{ppm} \mathrm{Th}$, and green albite $20 \mathrm{ppm} \mathrm{U}$ and $45 \mathrm{ppm}$ Th. These minerals exhibit black submicroscopic powder in their microfractures. According to Leonova et al. (1958), uranium occurs in quartz and feldspars as discrete submicroscopic uranium minerals or as scattered uranium molecules, which are absorbed by crystal planes or deformed crystal lattices.

\section{References}

Bernas, B. (1968) A new method for decomposition and comprehensive analysis of silicates by atomic absorption spectrometry. Anal. Chem. 40: $1682-1686$.

Beus, A. A. (1966) Geochemistry of beryllium and genetic types of beryllium deposits. W. H. Freeman and Company, San Francisco, pp. $331-332$.
Bräuer, H. (1970) Spurenelementgehalte in Granitischen Gesteinen des Thüringer Waldes und des Erzgebirges. Freiberger Forschungshefte C 259: $85-139$.

Erämetsä, O., Nieminen, K. and Niinistö, L. (1973) Two transparent beryl varieties from the Kaatiala pegmatite, Finland. Bull. Geol. Soc. Finland 45: $125-130$. 
Flanagan, F. J. (1973) 1972 values for international geochemical reference samples. Geochim. Cosmochim. Acta 37: 1189-1200.

Haapala, I., Siivola, J. and Löfgren, A. (1967) On the Haapaluoma Sc-bearing columbite and its inclusions. C. R. Soc. Géol. Finlande 34: 95100.

Holmberg, H. J. (1857) Mineralogische Wegweiser durch Finnland. Bidrag till Finlands Naturkännedom, Etnografi och Statistik. Första Häftet, pp. $3-76$.

Hutchison, C. S. (1974) Laboratory handbook of petrographic techniques, John Wiley \& Sons, New York, pp. $419-425$.

Laitakari, A. (1914) Kuortaneen pegmatiti. Manuscript, Department of Geology and Mineralogy, University of Helsinki.

Lappalainen, R. and Neuvonen, K. J. (1968) Trace elements in some Finnish pegmatitic potassium feldspars. Bull. Geol. Soc. Finland 40: 59-64.

Leonova, L. L. and Tauson, L. V. (1958) The distribution of uranium in the minerals of Caledonian granitoids of the Susamyr batholith (Central Tien Shan). Geochemistry (Int.) 1958: 815-826.

Nance, W. B. and Taylor, S. R. (1976) Rare earth element patterns and crystal evolution - I. Australian post-archean sedimentary rocks. Geochim. Cosmochim. Acta 40: 1539-1551.

Neuvonen, K. J. (1960) The pegmatites of Kaatiala. Int. Geol. Congr., 21st Sess: $50-51$.

Nieminen, K. and Yliruokanen, I. (1974) Trace element analysis of granitic and radioactive rocks by spark source mass spectrometry with electrical detection. Bull. Geol. Soc. Finland 46: 167-176.
Norton, J. J., Page, L. R. and Brobst, D. A. (1962) Geology of the Hugo pegmatite, Keystone, South Dakota. U.S. Geol. Surv. Prof. Pap. 279-B: $49-126$.

Norton, J. J. (1970) Composition of a pegmatite, Keystone, South Dakota. Am. Mineral. 55: 981-1002.

Oftedal, I. (1964) Distribution of boron in pegmatite. Norsk Geol. Tidskr. 44: 217-225.

Povarennykh, A. S. (1972) The role of crystallochemical factors. Int. Geol. Congr. 24th Sess., Sect. 10: 85-92.

Stavrov, O. D. and Khitrov, V. G. (1962) A possible geochemical relation of cesium and boron. Geochemistry (Int.) 1962: 53-61.

Tavela, M. (1949) Selostus Kuortaneen Vilppulan maasälpäpegmaattien geologiasta. Unpublished report to Suomen Mineraali Oy.

Taylor, S. R. (1964) Abundance of chemical elements in the continental crust: a new table. Geochim. Cosmochim. Acta 28: 1273-1285.

Taylor, S. R. (1965) Geochemical analysis by spark source mass spectrography. Geochim. Cosmochim. Acta 29: 1243-1261.

Taylor, S. R. (1968) Geochemistry of andesites. Pp. $559-585$ in Origin and distribution of the elements; ed. by L. H. Ahrens. Pergamon Press, Oxford.

Volborth, A. (1952) Alavuden N-osan ja Kuortaneen S-osan geologiaa (karttaselostus). Kaatialan ja Vilppulan pegmatiitit. Radioaktiivisuus. Lausunto ja työselostus v. 1951 suoritetuista tutkimuksista). Unpublished report to Suomen Mineraali Oy.

Manuscript received, January 13, 1978. 